\title{
Finite Unions of $D$-Spaces and Applications of Nearly Good Relation
}

\author{
Xin Zhang, ${ }^{1}$ Hongfeng Guo, ${ }^{1}$ and Yuming $X u^{2}$ \\ ${ }^{1}$ School of Mathematics and Quantitative Economics, Shandong University of Finance and Economics, \\ Jinan, Shandong 250014, China \\ ${ }^{2}$ School of Mathematics, Shandong University, Jinan, Shandong 250100, China
}

Correspondence should be addressed to Hongfeng Guo; guohongfeng@sdufe.edu.cn

Received 14 January 2013; Accepted 7 April 2013

Academic Editor: Fuyi Xu

Copyright (C) 2013 Xin Zhang et al. This is an open access article distributed under the Creative Commons Attribution License, which permits unrestricted use, distribution, and reproduction in any medium, provided the original work is properly cited.

\begin{abstract}
Some results are obtained on finite unions of $D$-spaces. It is proved that if a space is the union of finitely many locally compact $D$ subspaces, then it is a $D$-space. It follows that a space is a $D$-space if it is the union of finitely many locally compact submetacompact subspaces. And a space is a $D$-space if it is the union of a $D$-subspace with a locally compact $D$-subspace. This partially answers one problem raised by Arhangel'skii. At last, some examples are given to exhibit the applications of nearly good relation to discover D-classes.
\end{abstract}

\section{Introduction}

The concept of $D$-spaces was introduced by van Douwen and Pfeffer in [1]. It is well known that the extent coincides with the Lindelöf number in a $D$-space. Moreover, every countably compact $D$-space is compact and every $D$-space with the countable extent is Lindelöf. These facts make it helpful in studying covering properties. Some interesting work on $D$ spaces has been done by many topologists, especially by Arhangel'skii and Buzyakova (see [2-5]), Gruenhage (see [6]), Peng (see [7-9]), Fleissner and Stanley (see [10]), and Soukup (see [11])

Among the topics on $D$-spaces, the addition theorems occupy an important role. It has been an interesting subject, especially since Arhangel'skii raised the problem in $[2,3]$ whether the union of two $D$-subspaces is also $D$. In Section 3, we mainly consider the problem in locally compact spaces and give a partial answer by showing that a space is $D$ provided that it is the union of a $D$-subspace with a locally compact $D$-subspace. Besides, we obtain that if a space is the union of finitely many locally compact $D$-subspaces, then it is a $D$-space. With its help, it is shown that a space is a $D$-space if it is the union of finitely many locally compact submetacompact subspaces.
Another important task in studying $D$-spaces is to discover typical $D$-classes. The method is a key to do this work, and hence some methods and related concepts emerged during the process. Among them, we believe that the concept of nearly good relation is an important one, which was introduced in [6] by Gruenhage and helped to obtain that any space satisfying open $G$ is $D$. Unfortunately, the concept did not attract much attention, and hence it is difficult to find other results based on the construction of nearly good relations. In fact, it can help us discover some $D$-classes easily. In Section 4, we exhibit this with some examples. Moreover, we hope that our work will interest others in studying $D$ spaces and related open problems.

All spaces are assumed to be $T_{1}$-spaces.

\section{Definitions}

For the purpose of convenience, we recall the following definitions.

Definition 1. A neighborhood assignment is a mapping from a space to its topology. A space is called a $D$-space if, for every neighborhood assignment $\phi$ on $X$, there exists a closed 
discrete subset $A$ of $X$ such that the family $\phi(A)=\{\phi(x): x \in$ A) covers $X$.

Definition 2. A space is locally compact if each point in $X$ has a compact neighborhood.

Definition 3. A space is locally $D$ if each point in $X$ has a neighborhood which is a $D$-space.

In 1965, Worrell Jr. and Wicke in [12] introduced the class of $\theta$-refinable spaces. Since this class generalizes paracompact spaces, metacompact spaces, and submetacompact spaces, Junnila suggested in [13] that this class be renamed submetacompact spaces; we use this name in the following.

Definition 4. A sequence $\left\langle\mathscr{L}_{n}\right\rangle$ of covers of a space $X$ is $\theta$ sequence if, for every $x \in X$, there exists some $n \in N$ such that the family $\mathscr{L}_{n}$ is point finite at $x$. A space $X$ is called submetacompact, if every open cover of $X$ has a $\theta$-sequence of open refinement.

Note that, for a set $A$, define $[A]^{<\omega}=\{H \subset A:|H|<\omega\}$.

Definition 5 (see [7]). A relation $R$ on a space $X$ (resp., from $X$ to $[X]^{<\omega}$ ) is nearly good if $x \in \bar{A}$ implies $x R y$ for some $y \in A$ (resp., $x R \tilde{y}$ for some $\tilde{y} \in[A]^{<\omega}$ ).

Definition 6 (see [6]). Given a neighborhood assignment $\phi$ on $X$, a subset $Z$ of $X$ is $\phi$-close if $x, x^{\prime} \in Z \Rightarrow x \in \phi\left(x^{\prime}\right)$ (equivalently, $Z \subset \phi(x)$ for every $x \in Z$ ).

Definition 7 (see [6]). A family $\mathscr{L}$ of subsets of a space $X$ is point-countably expandable if there exists an open family $\left\{G_{L}\right.$ : $L \in \mathscr{L}\}$ such that $L \subset G_{L}$ for each $L \in \mathscr{L}$ and $\{L \in \mathscr{L}: x \in$ $\left.G_{L}\right\}$ for each $x \in X$.

Definition 8 (see [14]). A topological space $(X, \mathscr{T})$ is $t$ metrizable if there exists a metrizable topology $\pi$ on $X$ with $\tau \subset \pi$ and an assignment $H \mapsto J_{H}$ from $[X]^{<\omega}$ to $[X]^{<\omega}$ such that

$$
\bar{A}^{\mathscr{T}} \subset{\overline{\bigcup_{H \in[A]^{<\omega}} J_{H}}}^{\pi} \text { for every } A \subset X .
$$

Definition 9 (see [14]). A cover $\mathscr{L}$ of a topological space $X$ is thick if it satisfies the following condition.

One can assign $\mathscr{L}(H) \in \mathscr{L}^{<\omega}$ and $L(H)=\bigcup \mathscr{L}(H)$ to each $H \in[X]^{<\omega}$ so that

$$
\bar{A} \subset \bigcup\left\{L(H): H \in[A]^{<\omega}\right\} \quad \text { for every } A \subset X .
$$

Remark 10. In a $T_{1}$-space, the assignment " $\mathscr{L}(H) \in \mathscr{L}^{<\omega}$ " in the previous condition can be weakened to " $\mathscr{L}(H) \in \mathscr{L}^{\leq \omega}$ " [14, Lemma 2.1]. Hence if $(X, \mathscr{T})$ is a $T_{1}$-space, it is enough that the assignment $H \rightarrow J_{H}$ is from $[X]^{<\omega}$ to $[X]^{\leq \omega}$ in the definition of $t$-metrizable space.

Definition 11 (see [15]). A family $\mathscr{P}=\bigcup_{x \in X} \mathscr{P}_{x}$ of subsets of $X$ is a weak base of $X$, if the following conditions holds. (a) For every $x \in X, x \in \cap \mathscr{P}_{x}$.

(b) If $U, V \in \mathscr{P}_{x}$, there exists $W \in \mathscr{P}_{x}$ such that $W \subset$ $U \cap V$.

(c) A set $U$ is open in $X$ if and only if, for every $x \in U$, there exists $P \in \mathscr{P}_{x}$ such that $P \subset U$.

In the following two sections, we denote by $\bar{A}$ the closure of $A$ in the whole space and by $\mathrm{Cl}_{Y} A$ the closure of a set $A$ in the space $Y$. Besides, denote by $\mathbb{N}$ the set of all positive natural numbers.

About other terminologies and notations that are omitted here, please refer to [16].

\section{Finite Union of Locally Compact $D$-Spaces}

Theorem 12. If a space $X$ is the union of finitely many locally compact D-subspaces, then it is a D-space.

Proof. Suppose that $X=\bigcup_{i=1}^{n} X_{i}$, where each $X_{i}$ is a locally compact $D$-space.

To prove that $X$ is a $D$-space, let $\phi$ be a neighborhood assignment on $X$. We prove inductively and suppose that $X=X_{1} \cup X_{2}$ firstly.

Claim 1. $X_{1}$ is open in $\overline{X_{1}}$.

Proof of Claim 1. Denote $Y=\overline{X_{1}}$, and take an $x \in X_{1}$. Since $X_{1}$ is locally compact, let $U$ be an open neighborhood of $x$ such that $\mathrm{Cl}_{X_{1}} U$ is compact in $X_{1}$. Choose an open subset $V$ of $Y$ with $U=V \cap X_{1}$. Then $\mathrm{Cl}_{Y}\left(V \cap X_{1}\right) \cap X_{1}=\mathrm{Cl}_{Y} U \cap X_{1}=\mathrm{Cl}_{X_{1}} U$. It follows that $\mathrm{Cl}_{Y}\left(V \cap X_{1}\right) \cap X_{1}$ is compact and hence closed in $Y$. Moreover, it contains $V \cap X_{1}$ and thus contains $\mathrm{Cl}_{Y}\left(V \cap X_{1}\right)$. Then $\mathrm{Cl}_{Y}\left(V \cap X_{1}\right) \subset X_{1}$, and hence $\left(\mathrm{Cl}_{Y} X_{1}\right) \cap V \subset X_{1}$. It follows that $\left(\mathrm{Cl}_{Y} X_{1}\right) \cap V=V$ is an open neighborhood of $x$ in $Y$. Therefore, $X_{1}$ is open in $Y$.

Claim 2. The set $Z=\overline{X_{1}} \backslash X_{1}$ is closed in $X$.

Proof of Claim 2. Take an $x \in X \backslash Z$. If $x \in X_{1}$, then $V=\left(X \backslash \overline{X_{1}}\right) \cup X_{1}$ is an open neighborhood in $X$ such that $V \cap Z=\emptyset$. If $x \in X_{2} \backslash X_{1}$, then $x \in X_{2} \backslash \overline{X_{1}}$, and assume on the contrary that $x \in \bar{Z}$, which would follow that every neighborhood intersects $Z$ and thus intersects $X_{1}$, a contradiction with the fact $x \notin Z \subset \overline{X_{1}}$.

As a closed subspace of the $D$-space $X_{2}$, the space $Z$ is a $D$-space. Then there exists a closed discrete subset $D_{1}$ of $Z$, such that $\cup \phi\left(D_{1}\right) \supset Z$. Moreover, by Claim 2, Z is closed in $X$, so the set $D_{1}$ is also closed and discrete in $X$.

The set $H=X_{1} \backslash \cup \phi\left(D_{1}\right)$ is closed in $X$. Indeed, for any $x \notin H$, we have that $x \in \cup \phi\left(D_{1}\right)$ or $x \in X_{2} \backslash \cup \phi\left(D_{1}\right)$. If $x \in$ $\cup \phi\left(D_{1}\right)$, then $\cup \phi\left(D_{1}\right)$ is an open neighborhood of $x$ missing $H$; if $x \in X_{2} \backslash \cup \phi\left(D_{1}\right)$, then $x \notin X_{1}$, and hence $x \notin \cup \phi\left(D_{1}\right) \cup$ $X_{1} \supset Z \cup X_{1}=\bar{X}_{1}$, so $X \backslash \overline{X_{1}}$ is an open neighborhood of $x$ missing $H$.

As a closed subspace of $X_{1}, H$ is also a $D$-space. There exists a closed discrete subset $D_{2}$ in $H$, and thus in $X$, such that $\cup \phi\left(D_{2}\right) \supset H$. 
Clearly, the set $L=X \backslash \cup \phi\left(D_{1} \cup D_{2}\right)$ is closed in $X$ and contained in $X_{2}$. Then there exists a closed discrete subset $D_{3}$ in $L$, and thus in $X$, such that $\cup \phi\left(D_{3}\right) \supset L$.

It is trivial to check that $D=D_{1} \cup D_{2} \cup D_{3}$ is closed and discrete in $X$. Moreover, $\phi(D)$ is a cover of $X$ since $X=Z \cup$ $H \cup L$. Therefore, $X$ is a $D$-space, and we complete the proof for the case $n=2$.

For the case $n>2$, assume inductively that $\bigcup_{i=1}^{n-1} X_{i}$ is a $D$-subspace. Since the subspace $X_{n}$ is locally compact and open in its closure, with a similar construction as foregoing process, we can obtain a closed and discrete subset $E$ of $X$ such that $\phi(E)$ covers $X$. And thus $X$ is a $D$-space.

As a corollary of Theorem 12, we have the following consequence.

Corollary 13. Suppose that $X=\bigcup_{i=1}^{n} X_{i}$, where each $X_{i}$ is a submetacompact locally compact subspace. Then $X$ is a $D$ space.

Proof. Since compact space is $D$ and $X_{i}$ is locally compact for any $1 \leq i \leq n$, then every point of $X_{i}$ has a neighborhood which is $D$-subset; that is, the space $X_{i}$ is locally $D$. Moreover, because every locally submetacompact $D$-space is $D[16$, Theorem 5.10], each $X_{i}$ is a $D$-space. By Theorem 12 , as the union of finitely many locally compact $D$-spaces, the space $X$ is a $D$-space.

In fact, we see from the proof of Theorem 12 that, when $X=X_{1} \cup X_{2}$, the result can be obtained even only $X_{1}$ or $X_{2}$ is locally compact. So we have the following result, which is a partial answer to the problem whether a space is a $D$-space when the space is the union of two $D$-subspaces [3, Problem $1.1]$.

Theorem 14. Suppose that $X=X_{1} \cup X_{2}$, where $X_{1}$ and $X_{2}$ are all $D$-subspace and one of them is locally compact. Then $X$ is a D-space.

\section{Applications of Nearly Good Relation in Discovering $D$-Classes}

The following result presents us a method to discover $D$ spaces and we will show its use in this section with some examples. And we hope it will remind others with the use of nearly good relation in the study of $D$-spaces.

Proposition 15 (see [6]). Let $\phi$ be a neighborhood assignment on $X$. Suppose that there is a nearly good relation $R$ on $X$ (or from $X$ to $[X]^{<\omega}$ ) such that for any $y \in X$ (or $H \in[X]^{<\omega}$ ), $R^{-1}(y) \backslash \phi(y)$ (or $\left.R^{-1}(H) \backslash \cup \phi(H)\right)$ is the countable union of $\phi$-close sets. Then there is a closed discrete set $D$ such that $\cup \phi(D)=X$.

It is well known that every space with countable base is a $D$-space. In this section, we mainly show that some general properties can also imply $D$.

Firstly, the following result shows that many spaces with point-countable networks have $D$-property.
Proposition 16. Every space with a point-countably expandable network is a D-space.

Proof. Assume that $X$ has a point-countably expandable network $\mathscr{L}$ and the open family $\mathscr{G}=\left\{G_{L}: L \in \mathscr{L}\right\}$ satisfies that $L \subset G_{L}$ for each $L \in \mathscr{L}$ and $\left\{L \in \mathscr{L}: x \in G_{L}\right\}$ is countable for each $x \in X$.

To show that $X$ is a $D$-space, let $\phi$ be an arbitrary neighborhood assignment on $X$. Define a relation on $X$ as follows:

$$
x R y \Longleftrightarrow \exists L \in \mathscr{L}, \quad \text { such that } x \in L \subset \phi(x), y \in G_{L} .
$$

To show that $R$ is nearly good, let $A \subset X$, and let $x \in \bar{A}$. Since $\mathscr{L}$ is a network of $x$, there exists $L \in \mathscr{L}$ such that $x \in L$. Then $G_{L}$ is an open neighborhood of $x$, and hence $G_{L} \cap A \neq \emptyset$. Then there exists $y \in G_{L} \cap A$. It follows that $x R y$ and $R$ are nearly good.

For each $L \in \mathscr{L}$, let $C(L)=\{x: x \in L \subset \phi(x)\}$. Then for every $x \in C(L)$, we have that $\phi(x) \supset C(L)$; that is, $C(L)$ is a $\phi$ close set. By the definition of the relation $R$, it is easy to check that $R^{-1}(y)=\bigcup_{y \in G_{L}} C(L)$ is a countable union of $\phi$-close set. Hence by Proposition 15, there exists a closed discrete subset $D$ of $X$ such that $\bigcup \phi(D)=X$. And thus $X$ is a $D$-space.

In [14], a well-behaved class: $t$-metrizable spaces were introduced and then were proved in [17] to have $D$-property. Besides, as another good generalization of point-countable base, the point-countable weak base also implies $D$-property shown in [18]. However, the proofs of both results are very complicated. With the help of constructions of nearly good relations, we can prove them much easier.

Proposition 17 (see [17]). Every t-metrizable space is a $D$ space hereditarily.

Proof. Suppose that $X$ is a $t$-metrizable space. Since every subspace of $X$ is $t$-metrizable (see the remark following [14, Theorem 3.4]), we only need to show that $X$ is a $D$-space.

By [14, Theorem 3.4], $X$ has a network $\mathscr{F}=\bigcup_{n \in \omega} \mathscr{F}_{n}$, where each $\mathscr{F}_{n}$ is a thick partition of $X$. Then for all $n \in \omega$ and $H \in[X]^{<\omega}$, let $\mathscr{F}_{n}(H) \in \mathscr{F}_{n}^{<\omega}$ and $F_{n}(H)=\cup \mathscr{F}_{n}(H)$ be such that $\bar{A} \subset \cup\left\{F_{n}(H): H \in[A]^{<\omega}\right\}$ for every $A \subset X$.

To show that $X$ is a $D$-space, let $\phi$ be an arbitrary neighborhood assignment on $X$. Define a relation from $X$ to $[X]^{<\omega}$ as follows:

$x R H \Longleftrightarrow \exists n \in \omega, F \in \mathscr{F}_{n}(H)$, such that $x \in F \subset \phi(x)$.

To show that $R$ is nearly good, let $A \subset X$, and let $x \in \bar{A}$. Since $\mathscr{F}$ is a network of $X$, there exist $m \in \omega$ and $F_{x} \in \mathscr{F}_{m}$ such that $x \in F_{x} \subset \phi(x)$.

We have that $\bar{A} \subset \bigcup\left\{F_{m}(H): H \in[A]^{<\omega}\right\}$, and it follows that there exists $J \in[A]^{<\omega}$ such that $x \in F_{m}(J)=\bigcup \mathscr{F}_{m}(J)$. Moreover, since $\mathscr{F}_{m}$ is a partition of $X$ and $x \in F_{x} \in \mathscr{F}_{m}$, then $F_{x} \in \mathscr{F}_{m}(J)$. Therefore such $m$ and $F_{x}$ witness that $x R H$. We have shown that $R$ is a nearly good relation. 
Fix $H \in[X]^{<\omega}$. For each $n \in \omega$ and $F \in \mathscr{F}_{n}(H)$, let $C(F)=\{x: x \in F \subset \phi(x)\}$. For every $x \in C(F)$, we have that $\phi(x) \supset C(F)$, and it follows that $C(F)$ is a $\phi$-close set. By the definition of the relation $R$, it is easy to check that $R^{-1}(H)=\bigcup_{n \in \omega} \bigcup_{F \in \mathscr{F}_{n}(H)} C(F)$.

By Proposition 15, there exists a closed discrete subset $D$ of $X$ such that $\bigcup \phi(D)=X$. We have shown that $X$ is a $D$ space.

Proposition 18 (see [18]). Every space with a point-countable weak base is a D-space.

Proof. Suppose that $X$ has a weak base $\mathscr{P}=\left\{\mathscr{P}_{x}: x \in X\right\}$ such that $\{P \in \mathscr{P}: x \in P\}$ is countable for every $x \in X$.

We call a finite family $\left\{P_{x_{i}}: 1 \leq i \leq n\right\}$ a chain of length $n$ from $x$ to $y$ if, for every $1 \leq i \leq n, x_{i+1} \in P_{x_{i}}$ for some $P_{x_{i}} \in \mathscr{P}_{x_{i}}$ where we denote $x=x_{1}$ and $y=x_{n+1}$.

To show that $X$ is a $D$-space, let $\phi$ be an arbitrary neighborhood assignment on $X$ and define a relation on $X$ in the following way:

$$
\begin{array}{r}
x R y \Longleftrightarrow \exists \text { a chain }\left\{P_{x_{i}}: 1 \leq i \leq n\right\} \text { from } x \text { to } y, \\
\text { where } x_{1}=x .
\end{array}
$$

To show that $R$ is nearly good, let $A \subset X$ and $x \in \bar{A}$. We construct a neighborhood of $x$ as follows.

Step 1. Take a $P_{x} \in \mathscr{P}_{x}$.

Step 2. For every $a \in P_{x}$ taken in Step 1, take a $P_{a} \in \mathscr{P}_{a}$.

Inductively, we take other sets in $\mathscr{P}$ in following steps. Assume that Step $n-1$ has been finished, and now we go to Step $n$.

Step $n$. For every $P$ taken in Step $n-1$ and every $b \in P$, take a $P_{b} \in \mathscr{P}_{b}$.

Denote by $U$ the union of the set $P$ taken in all steps. Then $U$ is a open neighborhood of $x$. Indeed, for every $z \in U$, there must exist an $n \in \mathbb{N}$ and $P$ taken at Step $n$ such that $z \in P$; then at Step $n+1$, one $P_{z} \in \mathscr{P}_{z}$ will be taken, and thus $P_{z} \subset U$.

Since $x \in \bar{A}$ and $U$ is an open neighborhood of $x$, then there exists $y \in A$ such that $y \in U$. It follows from the definition of $R$ that $x R y$. Thereby, the relation $R$ is nearly good.

Since $\mathscr{P}$ is point countable, then for each $y \in X$ and $n \in$ $\mathbb{N}$, the set $L_{n}(y)=\{x \in X: x R y$, and the length from $x$ to $y$ is $n$ \} is countable. It follows that $R^{-1}(y)=\bigcup_{n \in \mathbb{N}} L_{n}(y)$ is countable, and hence it is the union of countable union of $\phi$-close set.

\section{Acknowledgments}

Xin Zhang is supported by the Natural Science Foundation of Shandong Province Grants ZR2010AQ001 and ZR2010AQ012, Hongfeng Guo is supported by the Natural Science Foundation of China Grants 11026108 and 11061004 , and Yuming Xu is supported by the Natural Science Foundation of Shandong Province Grants ZR2010AM019, ZR2011AQ015, and 2012BSB01159.

\section{References}

[1] E. K. van Douwen and W. F. Pfeffer, "Some properties of the Sorgenfrey line and related spaces," Pacific Journal of Mathematics, vol. 81, no. 2, pp. 371-377, 1979.

[2] A. V. Arhangel'skii, "D-spaces and finite unions," Proceedings of the American Mathematical Society, vol. 132, no. 7, pp. 21632170, 2004.

[3] A. V. Arhangel'skii, " $D$-spaces and covering properties," Topology and Its Applications, vol. 146-147, pp. 437-449, 2005.

[4] A. V. Arhangel'skii and R. Z. Buzyakova, "Addition theorems and D-spaces," Commentationes Mathematicae Universitatis Carolinae, vol. 43, no. 4, pp. 653-663, 2002.

[5] R. Z. Buzyakova, "Hereditary D-property of function spaces over compacta," Proceedings of the American Mathematical Society, vol. 132, no. 11, pp. 3433-3439, 2004.

[6] G. Gruenhage, "A note on D-spaces," Topology and Its Applications, vol. 153, no. 13, pp. 2229-2240, 2006.

[7] L.-X. Peng, "The D-property of some Lindelöf spaces and related conclusions," Topology and Its Applications, vol. 154, no. 2, pp. 469-475, 2007.

[8] L.-X. Peng, "A note on D-spaces and infinite unions," Topology and Its Applications, vol. 154, no. 11, pp. 2223-2227, 2007.

[9] L.-X. Peng, "On spaces which are $D$, linearly $D$ and transitively D," Topology and Its Applications, vol. 157, no. 2, pp. 378-384, 2010.

[10] W. G. Fleissner and A. M. Stanley, "D-spaces," Topology and Its Applications, vol. 114, no. 3, pp. 261-271, 2001.

[11] D. T. Soukup, "Constructing $a D$, non- $D$-spaces," Topology and Its Applications, vol. 158, no. 10, pp. 1219-1225, 2011.

[12] J. M. Worrell Jr. and H. H. Wicke, "Characterizations of developable topological spaces," Canadian Journal of Mathematics, vol. 17, pp. 820-830, 1965

[13] H. Junnila, "On submeta compact spaces," Topology Proceedings, vol. 3, pp. 375-405, 1978.

[14] A. Dow, H. Junnila, and J. Pelant, "Coverings, networks and weak topologies," Mathematika, vol. 53, no. 2, pp. 287-320, 2006.

[15] A. V. Arkhangel'skii, "Mappings and spaces," Russian Mathematical Surveys, vol. 21, no. 4, pp. 115-162, 1966.

[16] R. Engelking, General Topology, Polish Scientific Publishers, Warsaw, Poland, 1977.

[17] H. Guo and H. Junnila, "D-spaces and thick covers," Topology and Its Applications, vol. 158, no. 16, pp. 2111-2121, 2011.

[18] D. K. Burke, "Weak-bases and D-spaces," Commentationes Mathematicae Universitatis Carolinae, vol. 48, no. 2, pp. 281-289, 2007. 


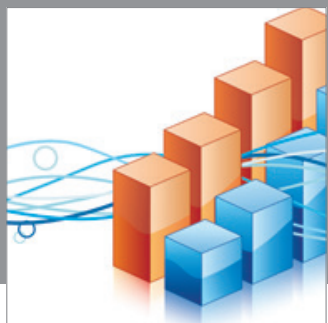

Advances in

Operations Research

mansans

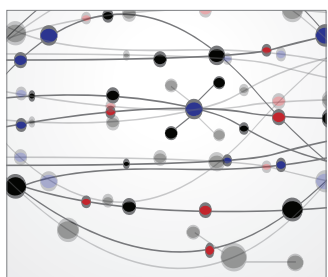

The Scientific World Journal
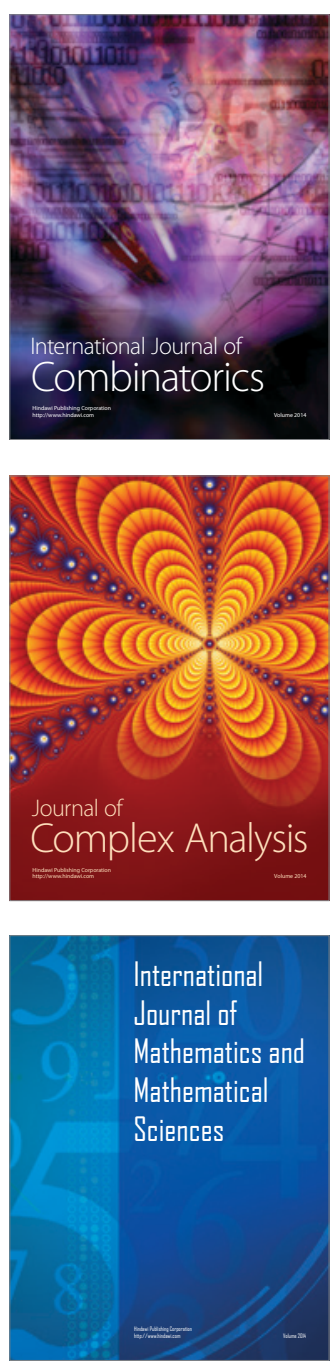
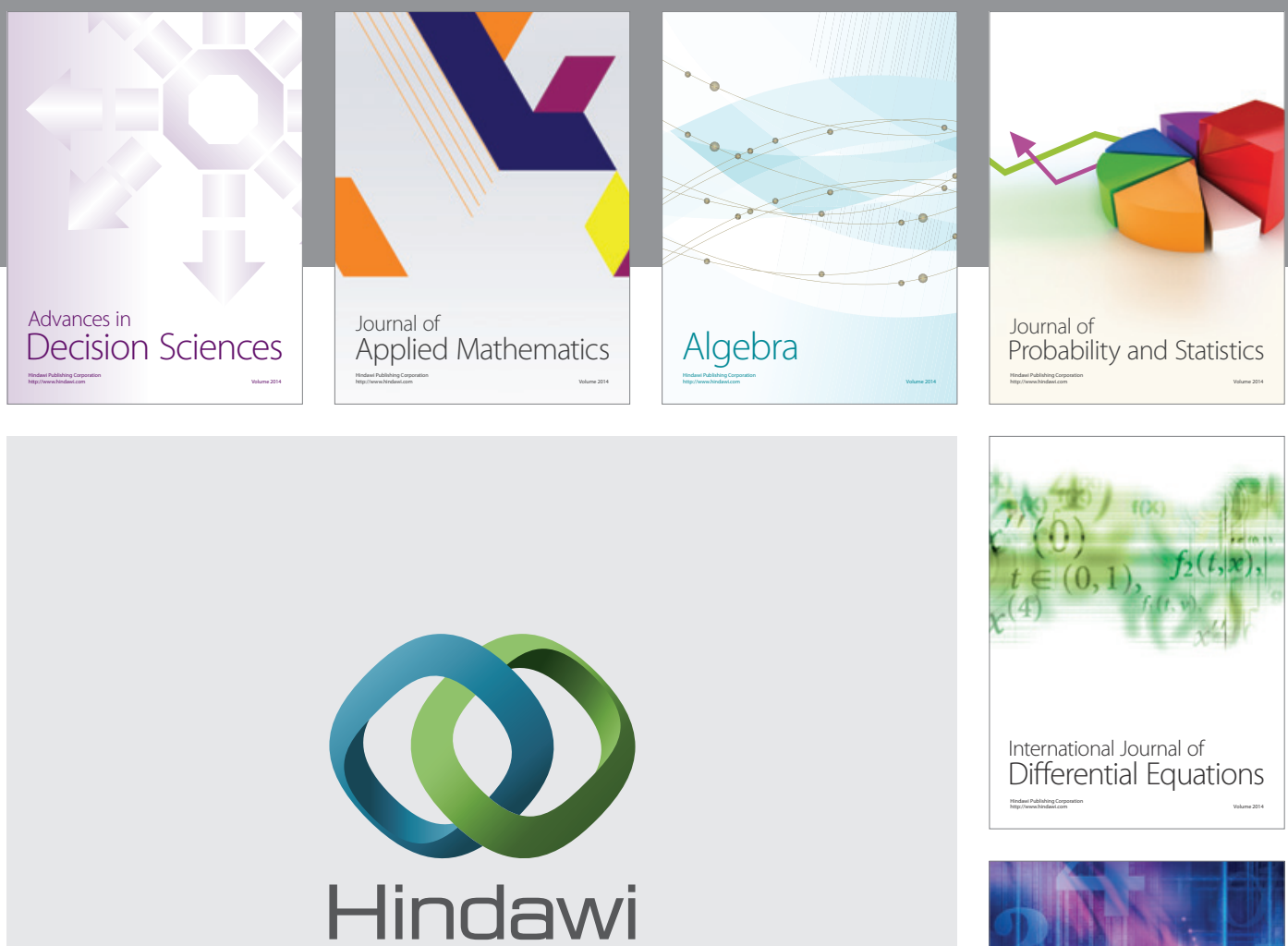

Submit your manuscripts at http://www.hindawi.com
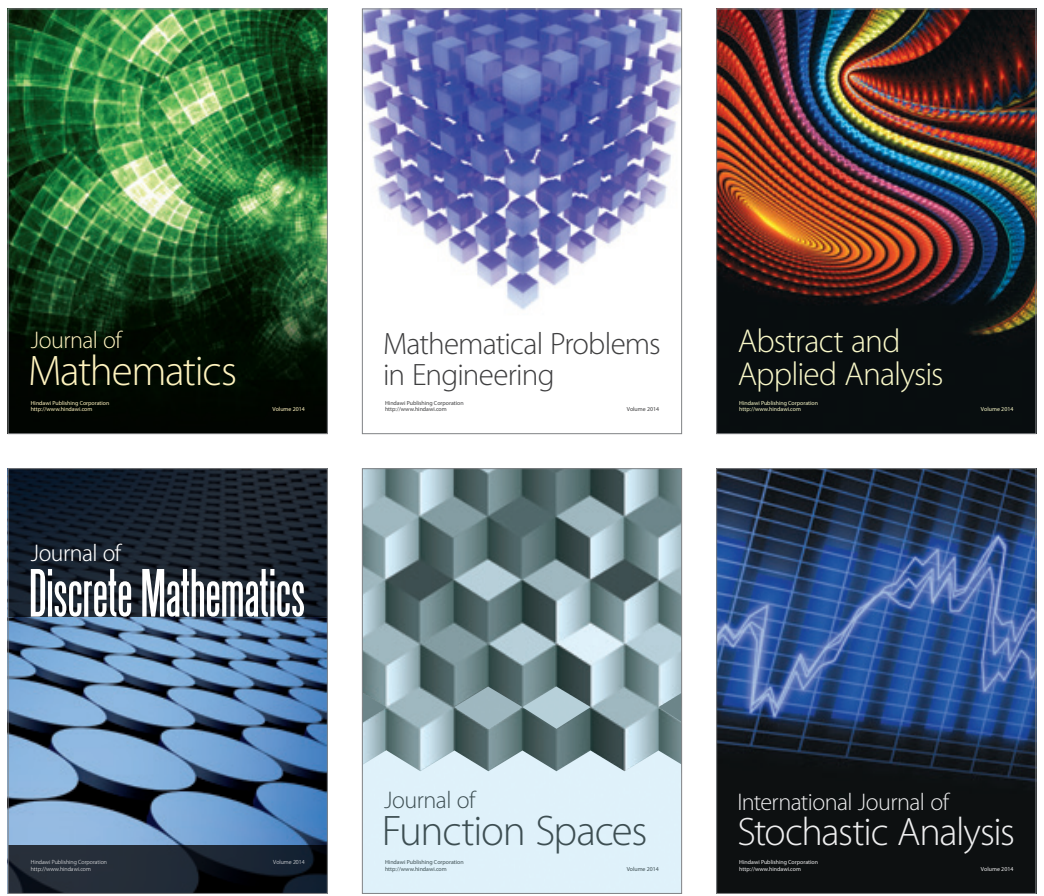

Journal of

Function Spaces

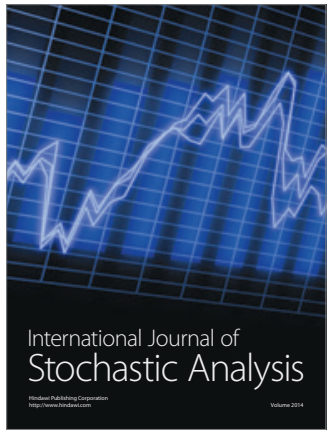

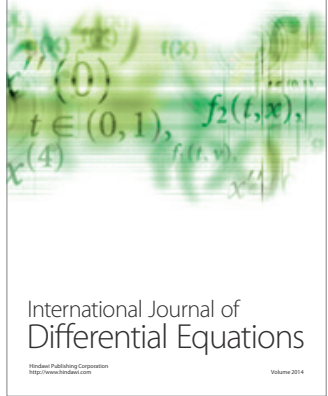
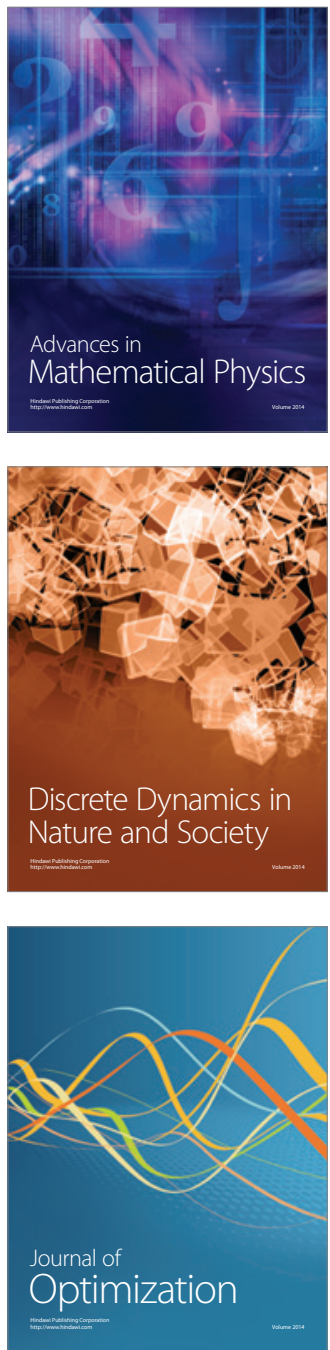